East African Medical Journal Vol. 80 No. 12 December 2003

INFECTIVITY OF PLASMODIUM FALCIPARUM GAMETOCYTES IN PATIENTS ATTENDING RURAL HEALTH CENTRES IN WESTERN KENYA L.C. Gouagna, PhD, Scientist, International Centre of Insect Physiology and Ecology (ICIPE), P.O. Box 30772, Nairobi, Kenya, B.A. Okech, PhD, Student. and E.W. Kabiru, PhD, Senior Lecturer, Department of Zoology, Kenyatta University, P.O. Box 43844 Nairobi, Kenya; Kenya Medical Research Institute, Nairobi, Kenya; G.F. Killeen, PhD, Scientist, Department of Public Health and Epidemiology. Swiss Tropical Institute, Socinstrasse 57. CH.4002, Basel, Switzerland; P. Obare, Technologist, International Centre of Insect Physiology and Ecology (ICIPE), P.O. Box 30772, Nairobi, Kenya; S. Ombonya, Medical Technologist, Mbita Health Center, Ministry of health, P.O. Box 50, Mbita, Suba District- Kenya, J.C. Beier, PhD, Scientist, School of Public Health and Tropical Medicine, Department of Tropical Medicine, Tulane University, 1430 Tulane Avenue, Louisiana 70112, USA; B.G.J. Knols, PhD. Scientist, Department of Entomology, Wageningen University Research Centre, P.O. Box 8031, 6700 EH, Wageningen, The Netherlands, J.I. Githure, PhD, Head of Human health Division, International Centre of Insect Physiology and Ecology (ICIPE), P.O. Box 30772, Nairobi, Kenya and G. Yan, PhD, Scientist, Department of Biological Sciences, State University of New York, Buffalo, NY 14260, USA

Request for reprints to: Dr. L. C. Gouagna, ICIPE, P. O. Box 30772, Nairobi, Kenya

\title{
INFECTIVITY OF PLASMODIUM FALCIPARUM GAMETOCYTES IN PATIENTS ATTENDING RURAL HEALTH CENTRES IN WESTERN KENYA
}

L. C. GOUAGNA, B. A. OKECH, E. W. KABIRU, G. F. KILlEEN, P. OBARE, S. OMBONYA, J. C. BIER, B. G. J. KNOLS, J. I. GITHURE and G. YAN

\begin{abstract}
Background: Experimentally studying the transmission of the malaria parasite and its regulating factors requires availability of human blood donors carrying infectious gametocytes. The difficulty of identifying gametocyte carriers from the community is often limited due to financial and human resources constraints. The available alternative is rural health centres where malaria patients go for treatment. In this study, the potential of recruiting volunteers and acquiring infectious blood for experimental infections from rural health centers in malaria endemic area was examined through routine patient diagnosis.

Objective: To examine the patients presenting at rural health centers for the potential to carry sexual stage malaria parasite and test their infectivity to Anopheles gambiae mosquitoes.

Setting: Mbita Health Centre, Mbita Town Ship, Suba District, western Kenya.

Methodology: Routine survey of all patients attending Mbita Health Centre with suspected malaria. Patients were examined for Plasmodium falciparum trophozoites and gametocytes. Gametocyte-positive volunteers were recruited for their potential to infect Anopheles mosquitoes via membrane feeding.

Results: Three thousand nine hundred and eighty seven patients were screened between May 2000 and April 2001. Plasmodium falciparum was the predominant parasite species and $P$. malariae being the only minor species, accounting for $0.9 \%$ of malaria cases. Clinical malaria varied with age and prevailed throughout the year with a slight seasonality. Gametocyte prevalence was low $(0.9-6.6 \%)$, and gametocyte densities were generally very low with a geometric mean of 39 gametocytes per $\mu$ l blood. Children aged $>5$ years constituted $67 \%$ of all gametocyte carriers. Only 22 volunteers with mean gametocytes density of 39.62 per $\mu \mathrm{l}$ blood (range: 16-112) were recruited for study of parasite infectiousness to laboratory-reared mosquitoes. Only two patients infected $1 \%$ of 1099 mosquitoes with one or two oocysts.

Conclusion: The low gametocyte densities or other possible host and vector related factors regulating infectivity of gametocyte carriers to mosquitoes may have caused the poor infections of mosquitoes. This study indicates that rural health centers in malaria-endemic areas may not be suitable for recruiting infectious gametocyte donors for studies of vector competence. They are suitable for passive clinical case surveillance and for evaluation of the effects of control measures.
\end{abstract}

\section{INTRODUCTION}

Human malaria is caused by specialized Plasmodium parasites that are adapted to propagate alternately in two very different hosts: Anopheles vector mosquitoes and human beings. Malaria parasites, $P$. falciparum in particular, are highly pathogenic in humans and cause an immense public health burden, especially in sub-Saharan Africa(1,2). Each year, millions of affected people attend clinics, health centres and hospitals throughout the tropics(3), placing a huge financial constraint upon both the individuals themselves and healthcare infrastructures that serve them $(4,5)$. The prevalence and incidence of malaria infection, morbidity and mortality is dictated by both the ecology of the mosquito vectors and the dynamics of infection within the human populations $(1,6,7)$. The progression of any malaria infection within a human host has two important possible outcomes: pathogenesis and transmission. Both the asexual parasite stages related to the pathogenicity 
(trophozoites and merozoites) and those responsible for transmissibility (gametocytes) can be routinely detected by microscopic examination of smeared blood samples at many health facilities. Experimentally studying the transmission of the malaria parasite and its regulating factors requires availability of human blood donors carrying infectious gametocytes(8-12). Health facilities could therefore represent sampling site through which gametocyte carriers may be selected for studies on human infectiousness. Several recent studies have demonstrated the infectivity of gametocyte carriers recruited from clinically ill subjects in Cameroon(8), Senegal(12), Gambia and Tanzania(13). These studies, have found high proportion of individuals carrying gametocytes either at recruitment or following treatment of patients previously assumed to be infected with $P$. falciparum.

Here we attempted to use the same sample design and reproduce features of gametocyte infectivity found elsewhere. We evaluated the feasibility of using rural health centres in Western Kenya-malaria endemic areas for recruiting gametocyte-carrying volunteers for vector competence studies. We examined the potential of volunteer patients with $P$. falciparum gametocytes to infect Anopheles gambiae mosquitoes via experimental membrane feedings. We chose Mbita health center, Suba District, western Kenya because the area is representative of the basin region of Lake Victoria with highest malaria transmission intensity in Kenya(14). The information on malaria parasite infectiousness is critical for understanding the mechanisms that regulate parasite transmission under natural conditions $(8,15)$ and for developing malaria control strategies aiming at blocking parasite transmission(16,17).

\section{MATERIALS AND METHODS}

Study area: The study was carried out at Mbita Health Centre in Mbita town, situated on the shore of Lake Victoria, in Suba District, Western Kenya. About 8,000 people live in Mbita area and most of them are resident fishermen but there are also some traditional farmers. Mbita community is constituted of several small housing groups adjacent to the lake because of the accessibility and fishing activities. Mbita also serves as a central trading hub for other parts of the district, especially Rusinga and Mfangano Islands where approximately 15,000 and 20,000 people subsist respectively, upon similar fishing and farming activities. Mbita Health Centre is the closest health facility to the International Centre for Insect Physiology and Ecology, Mbita Point Research and Training Centre, the base of an interdisciplinary programme investigating the ecology of malaria vectors and transmission in Suba district $(18,19)$. It therefore represents an ideal surveillance point from where the dynamics of human infection and infectiousness can be studied in parallel with other ongoing field studies of vector population dynamics.

Suba District is located between longitudes $34^{\circ} \mathrm{E}$ and $34^{\circ} 20$ "E and latitudes $0^{\circ} 20$ "S and $0^{\circ} 52$ "S. It includes 16 islands and covers an area of $1048 \mathrm{~km}^{2}$ exclusive of water surfaces. The area typically has two rainy seasons, the main rains extending from March to May and lesser rains in August to December. Annual rainfall ranges from $700 \mathrm{~mm}$ to 1,200 $\mathrm{mm}$. The area experiences high temperatures throughout the year ranging from $17^{\circ} \mathrm{C}$ to $34^{\circ} \mathrm{C}$. During the rainy season, there are ample breeding sites for An. gambiae, An. arbhiensis and $A n$. funestus mosquitoes. The area is considered malaria hyper- or holoendemic $(18,19)$.

Survey Protocol: The surveillance and recruitment procedures reported in the present study were approved by the ethical review committees of appropriate institutions. This protocol designated that gametocyte carriers detected at Mbita Health Centre could be recruited as potential blood donors on a voluntary basis, from patients between the age of 5 and 15 years, because this age group in western Kenya was shown to yield high levels of infection in experimentally infected mosquitoes(20).

Inclusion criteria for recruitment as blood donors were age and $P$. falciparum gametocyte carriage, whereas exclusion criteria included pregnancy, mixed infections and any symptoms indicating severe clinical malaria and other concomitant diseases requiring hospitalisation or follow-up. As in other studies from across Africa $(8,9,12,13,21)$, children under five years were excluded from recruitment because of concerns on the vulnerability of young children to delayed treatment during blood drawing. Potential donors were informed of the purpose and procedures of the study and potential benefits and risks associated with it and, if willing to participate, invited to sign an informed consent form, as reviewed and approved by ethical review committees. The survey and recruitment procedure was overseen by the resident clinical officer in Mbita Health Center, district laboratory technologist and medical officers.

Clinical examination and parasitological diagnosis: Between the beginning of May 2000 and the end of April 2001, all patients reporting suspected uncomplicated malaria were screened by microscopic examination of blood samples. Standard thick blood films from finger pricks, stained with $10 \%$ Giemsa were examined at $\mathrm{x} 1,000$ magnification. Trophozoite density was assessed and classified as not detectable, low density infection or high density infection, representing approximately $<50,50-2,000$ or $>2,000$ parasites/ $\mu \mathrm{l}$, based on calibration against the number of white blood cells typically observed per field(22). Because the initial objective was to detect only patients with falciparum gametocytes, the true level of asexual infections was not measured. Slides positive for either trophozoites or gametocytes were then searched for gametocytes in a further 100 fields and the number of $P$. falciparum gametocytes recorded. Later, gametocyte density, but not trophozoites was assessed through counting the number of parasites per 500 leucocytes. The counts were converted to numbers of gametocytes per $\mu 1$ blood by assuming a standard leukocyte count of 8,000 per $\mu 1$. Patient's history of symptoms, detailing clinical diagnosis and previous drug use before the screening formed a crucial part of the data collected during clinical survey.

Mosquito strain and rearing: A strain of An. gambiae sensu stricto from laboratory colony originating from Mbita itself, adapted to feed on Parafilm ${ }^{\circledR}$ membrane was used. Mosquitoes were reared in a semi-field adult insectary according to standard rearing procedures(23). Briefly, about 200 larvae in a $20 \times 15 \mathrm{~cm}$ tray were fed on $10 \%$ slurry of parts $2: 1$ of Tetramin Baby E® fish meal in a screen-walled greenhouse environment that closely resemble natural conditions. Pupae were collected and kept in $30 \times 30 \times 30$ meshed cages where emerging adults had access to $6 \%$ glucose solution. Batches of fifty to one hundred females, three days post emergence, 
were starved for 6-8 hours by removing both the water and glucose solution prior to the blood feeding.

Experimental infection of mosquitoes: Upon recruitment of gametocyte carriers and signing of the informed consent form, approximately 2 to $5 \mathrm{ml}$ of venous blood was drawn by the ministry of health clinical officer or health technician. This blood sample and portions thereof was then immediately fed to female mosquitoes using pre-warmed $\left(37^{\circ} \mathrm{C}\right)$ artificial membrane mini-feeders as previously described(8). They were allowed to feed for 15 minutes after which, unfed mosquitoes were removed from the cage and the remaining insects were maintained in the insectary under ambient conditions $\left(25-27^{\circ} \mathrm{C}, 60-80 \% \mathrm{RH}\right)$ with daily access to $6 \%$ glucose solution until dissection 7 days later to determine oocyst infections. Briefly, surviving mosquitoes $(>20$ per batch) were dissected on 7 days post-feeding and their midguts were stained with $2 \%$ mercurochrome in distilled water to facilitate examination for the presence and number of mature oocysts by light microscopy (10x). The ethical review committee of Kenya Medical Research Institute (KEMRI) approved all mosquito rearing and maintenance procedures, including feeding of human and rabbit blood. Statistical analysis: All statistical analyses were carried out using SPSS version 10.0.1 and Microsoft Excel 2000 for Windows, as described in the results section in relation to the outcomes examined. We compared parasitological indexes using age as grouping factor and for comparison between groups, Chi-square test was used to test hypothesis of difference between variables and to examine possible source of variation. The independent contrast between clinical symptoms, asexual positivity, previous treatment and gametocyte carriage was regressed using forward conditional selection procedures to obtain parameter values, which were tested by Chi-square to determine possible predictors and to select the most probable underlying predictor when faced with two or more covariants.

The ability of each experimental infection to produce mosquito infection was examined to determine whether or not selected carriers were infectious under laboratory conditions. Therefore, the outcomes of each infection included in the analysis were; (i) prevalence of positive infection and ii) intensity of oocyst in relation to gametocyte density in the blood samples.

\section{RESULTS}

Clinical malaria burden: Over the course of one-year survey, 3,987 patients were screened for malaria parasites, with more females $(55.5 \%)$ than males reporting to the clinic. The dominant age groups attending the clinic were young children, particularly infants, and young adults aged 16-30 years (Table 1). The vast majority of the patients attending Mbita clinic, the major health centre in the area came either from Mbita itself (54.2\%) or from nearby Rusinga Island (35.5\%).

Plasmodium falciparum was highly prevalent and parasites were identified in thick smears from almost half of the patients (Table 1). A total of 19 P. malariae cases $(0.9 \%)$ were observed, and among them 15 cases were mixed infections with $P$. falciparum. High $P$. falciparum prevalence was distributed across all age categories and over half of all children up to 14 years of age harbored patent infections, including very young infants (Table 1). Based on this distribution, we categorized all screened patients as young children ( $<5$ years), older children $(5-$ 15 years), young adults (16-30 years) and older adults (>30 years) for subsequent analyses.

Pearson correlation analysis showed that, on a monthly basis, the number of suspected malaria patients reporting to the clinic was directly correlated with the adjusted odd of high density infections $(\mathrm{P}=0.03$, correlation coefficient $=0.60$ ), indicating that malaria was responsible for a large proportion of the patient burden at Mbita Health Centre. In those reporting to the clinic, all parasitological indicators including asexual positivity (Figure la), high asexual parasite density (Figure lb) and gametocyte (Figure lc) was found to fluctuate seasonally. Interestingly, the number of patients reporting to the clinic with suspected clinical malaria followed a similar, if less pronounced seasonal pattern (Figure 1d). These parameters peaked briefly in June, following the heavy long rains and then more steadily between September and February, during which time the usual short rains extended, with essentially no continuous dry period, into the long rains (Figure 1 e).

Table 1

Age distribution of patients attending Mbita Health Centre, Suba District, Western Kenya

\begin{tabular}{lllll}
\hline & & $\begin{array}{l}\text { Proportion infected } \\
\text { with P. falciparum }\end{array}$ & $\begin{array}{l}\text { Proportion with } \\
\geq 2000 \text { P. falciparum } \\
\text { parasites per } \mu \text { l } \\
(\%)\end{array}$ & $\begin{array}{l}\text { Proportion with } \\
\text { gametocytes }(\%)\end{array}$ \\
Age Group & No. of Patients $(\%)$ & $(\%)$ & 12.8 & 6.6 \\
\hline 55 & $1,417(37.7)$ & 54.8 & 8.2 & 3.2 \\
$5-15$ & $635(16.9)$ & 57.3 & 2.8 & 0.9 \\
$16-30$ & $1,163(31.0)$ & 36.6 & 3.5 & 2.8 \\
$>30$ & $539(13.5)$ & 33.4 & 7.6 & 3.7 \\
\hline Overall & $3,754(100)$ & 46.5 & & \\
\hline
\end{tabular}


Table 2

The influence of age group upon patients' probability of having malaria infection at presentation ${ }^{a}$

\begin{tabular}{lll}
\hline & \multicolumn{2}{c}{ Odds Ratio $(95 \%$ confidence interval $)$} \\
Age Group & $\begin{array}{l}\text { Any detectable parasitaemia } \\
(>50 \text { per } \mu \mathrm{l} \text { blood })^{\mathrm{b}}\end{array}$ & $\begin{array}{l}\text { Heavy parasitaemia } \\
(>2000 \text { per } \mu \mathrm{blood})\end{array}$ \\
\hline$\leq 5$ & $2.42(1.97-2.98)$ & $4.03(2.49-6.54)$ \\
$6-15$ & $2.67(2.10-3.39)$ & $2.44(1.43-4.18)$ \\
$16-30$ & $1.15(0.93-1.43)$ & $0.80(0.45-1.49)$ \\
$>30$ & $1.00^{\mathrm{c}}$ & $1.00^{\mathrm{c}}$ \\
\hline
\end{tabular}

a Identified and quantified as significant risk factors by logistic regression using forward conditional selection. Cox and Snell $\mathrm{R}^{2}=0.040$ and $0.029,-2 \mathrm{Log}$ likelihood $=5032$ and 1911 for any parasitaemia and heavy parasitaemia models, respectively.

Hosmer and Lemshow goodness of fit probability $=1.00$ for both models.

$\mathrm{b}$ Limit of sensitivity for thick smear screening procedure. ${ }^{\mathrm{c}}$ Reference group; confidence intervals not applicable.

Table 3

The effects of age group and the presence of asexual malaria parasites upon patient's probability of carrying $P$. falciparum gametocytes at presentation

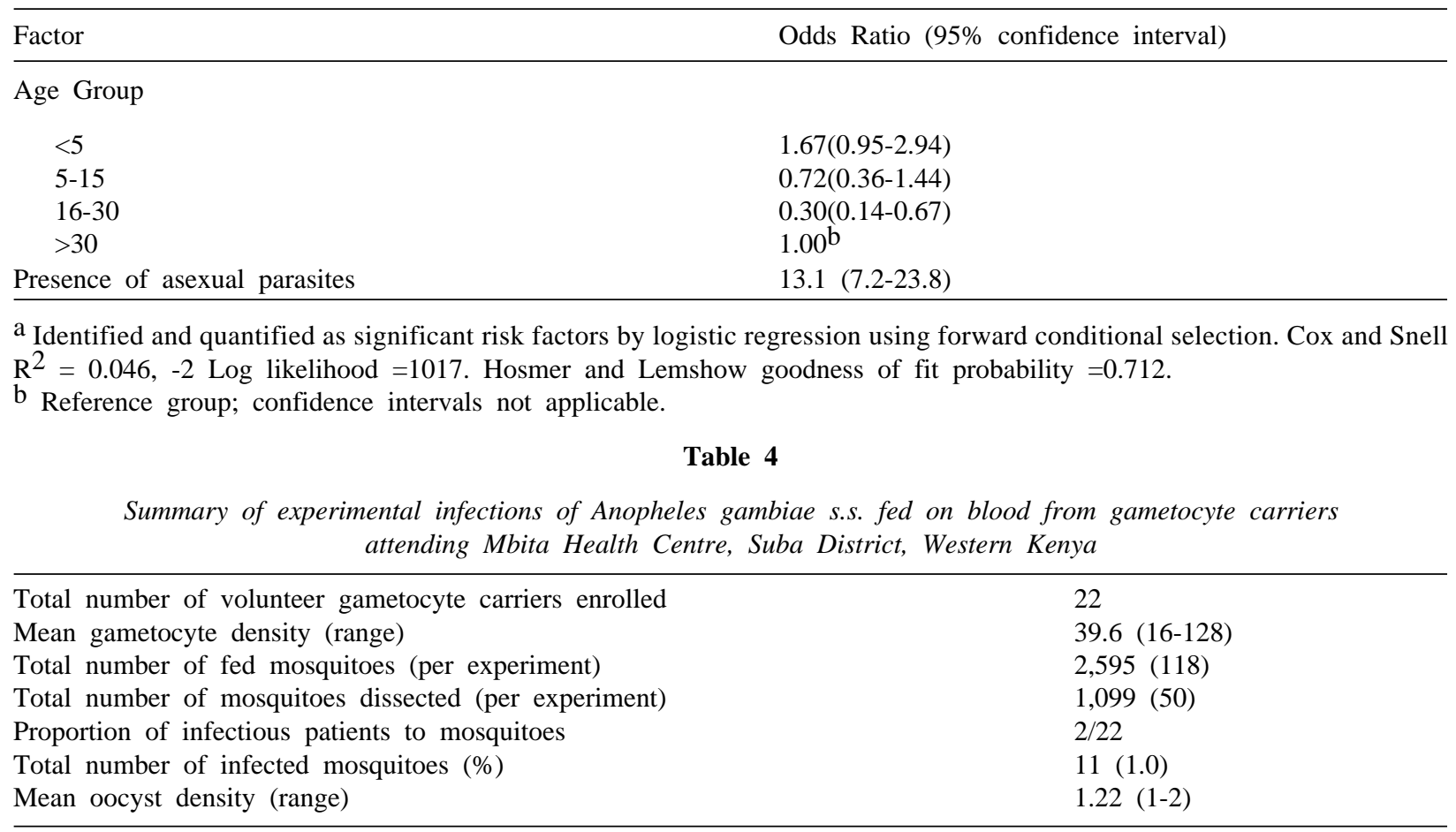

\section{Figure 1a}

Seasonality of malaria infection and patient load at Mbita Health Centre. The odds ratio for carrying any detectable infection

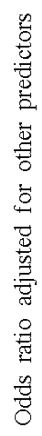

\section{Figure 1b}

Gametocytes

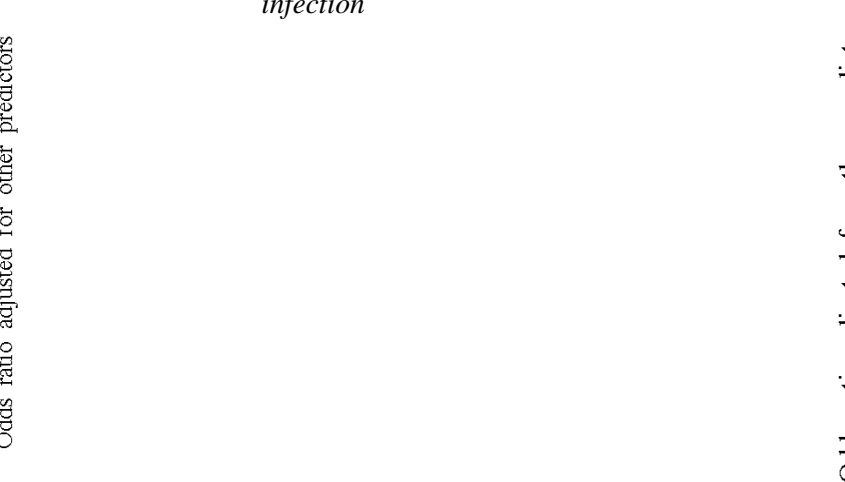

(A) High density infection 
Figure 1c

Were estimated by adding month as a factor to the logistic regression models, including the other predictive factors described in Tables 2 and 3. The total number of patients screened for malaria parasites

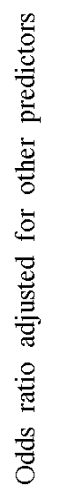

Figure 1d

Rainfall

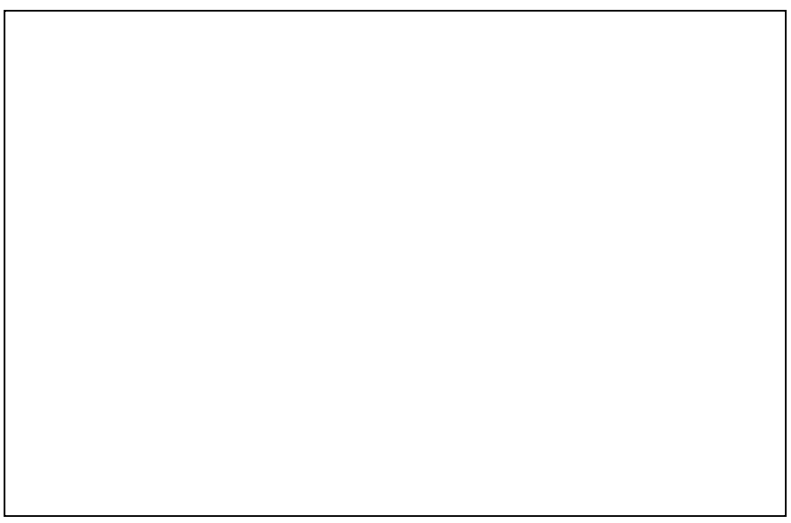

Figure 1e

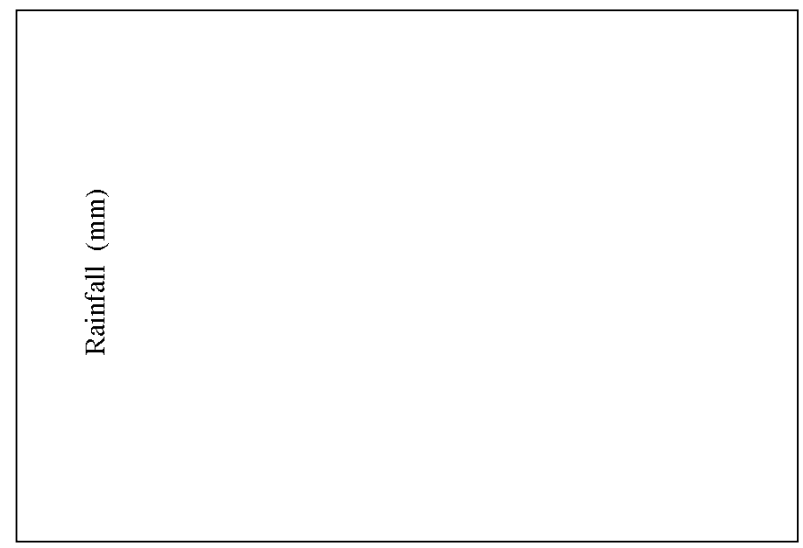

Each month (D) is also presented. Cox and Snell $\mathrm{R}^{2}=0.084$, 0.057 and 0.059 ; $-2 \log$ likelihood $=4857,1802$ and 966; Hosmer and Lemeshow goodness of fit probability $=0.572$, 0.502 and 0.522 for the logistic models described in A, B and $\mathrm{C}$ respectively.
Figure 2

Distribution of plasmodium falciparum gametocyte densities as a function of age

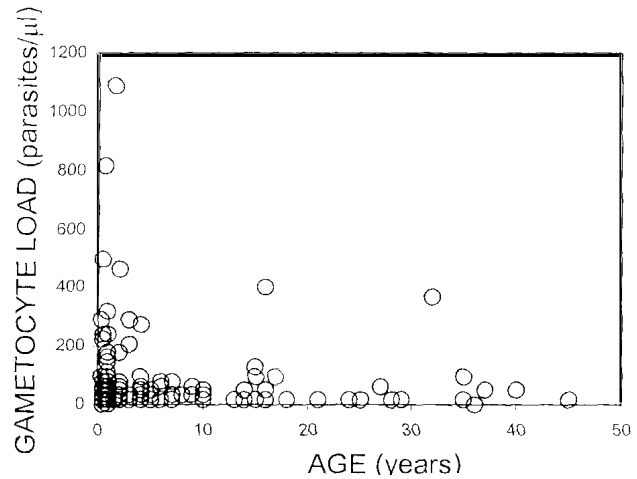

The prevalence of malaria parasites at detectable levels (>50 per $\mu \mathrm{l}$ ) was highest in children and high-density infections $(>2,000$ per $\mu \mathrm{l})$ were particularly found in the youngest age groups (Table 1). Only $2.9 \%$ of all patients reported having taken medication within two weeks prior to presentation, the most common treatments being pyrimethamine/sulphadoxine combinations such as Fansidar ${ }^{\circledR}$ and Metakelfin ${ }^{\circledR}(56$ patients), followed by chloroquine (Malaraquine $\AA$, Homaquine $\AA$ ), amodiaquine (Malaratab () ) and quinine $(34,22$ and three patients for each drug, respectively). Logistic regression using a forward conditional stepwise selection procedure revealed that age group but not previous treatment, or location of residence significantly influence the probability of patients having parasitaemias at detection threshold $\left(\mathrm{X}^{2}=149.0\right.$, d.f. $\left.=3, \mathrm{P}<0.001\right)$ or high densities $\left(\mathrm{X}^{2}=91.5\right.$, d.f. $\left.=3, \mathrm{P}<0.001\right)$. Consistent with Figure 1, children were more than twice likely to exhibit patent infections than adults (Table 2). Children aged $>5$ and 5-15 years were four and two fold more likely to harbor high density infections than adults, respectively.

Risk factors for gametocyte carriage: Only 3.7\% of the 3,987 patients were found to carry gametocytes, of which the majority $(63 \%)$ were young children (Tables 1 and 3). As expected, logistic regression, using forward conditional stepwise selection, determined that age group $\left(\mathrm{X}^{2}=35.0, \mathrm{df}=3, \mathrm{P}<0.001\right)$ and the presence of asexual parasites $\left(\mathrm{X}^{2}=63.9\right.$, df. $=1, \mathrm{P}$ $<0.001)$ but not previous treatment or parasite density significantly influenced the probability of patients being found to carry gametocytes at presentation (Table 3). Gametocyte densities were quite low overall with a geometric mean density of 39 (95\% confidence interval $(\mathrm{CI})=33$ - 46) parasites $/ \mu \mathrm{l}$, that decreased with age (Figure 2). Children aged $<5$ years constituted $67 \%$ of all gametocyte carriers. 
Feasibility of recruiting gametocyte carriers and infecting mosquitoes: In total, 1484 patients carrying $P$. falciparum gametocytes were detected during the study period. Most of them were either too young $(67 \%)$ or too old to be eligible under the approved protocols (target group 5-15 years). The target age group selected for recruitment represented less than $17 \%$ of the malaria patient load at the clinic and only $14.6 \%$ of all gametocyte carriers. Only 66 potential donors fulfilled both the inclusion and exclusion criteria of the protocol of whom only 22 were recruited (mean age: 10.2 years). The remaining $66.6 \%$ of eligible gametocyte carriers reported two or more clinical symptoms and refused to participate and/or were precluded from recruitment as potential donors for studies of human infectiousness and vector competence because of concerns of the vulnerability of these patients to treatment delay.

For enrolled gametocytes carriers in experimental membrane feeding study, the mean gametocytaemia was 39.62 gametocytes per $\mu$ l (range: $16-128 / \mu \mathrm{l}$ ). Out of over 2595 mosquitoes successfully fed on blood from the volunteer donors, approximately 1099 mosquitoes survived and were subsequently dissected for oocyst on day 7 post-feed. Table 4 shows the number of mosquitoes dissected per experiment and mean oocyst density per infected mosquito after feeding with blood from gametocyte carriers. On average, 50 mosquitoes per experiment were dissected. Only $1 \%$ $(11 / 1099)$ of the mosquitoes infected with 1.22 oocysts per infected mosquito, all infected mosquitoes having only one or two oocysts each.

\section{DISCUSSION}

As an important step towards studying determinants of vector competence, we assessed the gametocyte prevalence and density in patients reporting to a rural health clinic in Western Kenya. We found that the highest rate of clinical malaria cases tends to be concentrated in children less than five years of age. Infants one year old or less constituted the largest single age group reporting to the clinic and over $50 \%$ of these children were detectably infected with P. falciparum. This level of infection is considerably higher than nearby Siaya sites reported by other authors $(23,24)$. Paradoxically, clinical malaria was associated with a relatively low density of trophozoites. Although results from this clinic study are inappropriate to define community level endemicity, this low asexual densities and the associated low frequency of gametocyte carriage found in this endemic malaria area may result from high premunition, and possibly gametocyte-specific immunemediated clearance mechanisms(16).

The observation that antimalarial use prior to presentation had no influence on parasite infection intensity is somewhat alarming. Many of the patients reporting to the clinic may have had recrudescing, resistant infections. Although our results may be confounded by reluctance of patients to divulge information on their antimalarial drug uses, preliminary school surveys in this area suggest that over $10 \%$ of infected children may harbor parasites resistant to pyrimethamine/sulphadoxine(25). We have also noted widespread self-treatment and underdosing in this community. Studies on the coast of Kenya have shown pyrimethamine/sulphadoxine resistance is a rapidly evolving threat(26). The observed disease burden in outpatients attending the health centre in particular or in the population living in our study area in general, may be explained by a high level of inadequate automedication(27).

Gametocyte prevalence and density appear consistently low throughout the year, compared with other reports on community malaria surveillance $(9,10,20,28)$ and other surveys of clinical cases in Africa $(8,11,12)$. Our studies may have underestimated the size of the pool of infectious host donors since we had only sampled symptomatic infections; therefore it is possible that the range of volunteer subjects examined was not sufficient to accurately represent the infectiousness reservoir in human population in this area. However, the results may merit careful attention, including the potential of low level $P$. falciparum gametocytaemia in patients exposed to holoendemic malaria(23). Most gametocyte carriers may not seek medical care because the sexual stage of $P$. falciparum is not pyrogenic and is commonly seen in individuals with asexual parasite density below the level causing malaria symptoms $(30,31)$. In this hospital-based survey, it would be unethical to delay treatment to infected volunteers in order to monitor the extent of infectivity. Therefore, the vast majority of outpatients could not be recruited because of their age, severe clinical symptoms, and unwillingness to participate. Our sample could have been biased by excluding those with very severe symptoms. However, this is necessary when working with human subjects. It was assumed that the precision of our investigation reported here could have been improved by monitoring both the symptomatic and asymptomatic human groups.

Gametocyte production is thought to be typically associated with well-established infections and gametocyte formation appears after initial peak of asexual parasitaemia in response to a variety of stimuli such as drug pressure, erythrocyte lyses and host reactions(31-34). The gametocyte prevalence reported here are not representative of prevalence in human population of this area specifically because our samples were from a population from health clinic with malaria symptoms. Indeed, preliminary surveys in elementary schools in the same area indicate considerably higher levels of gametocytaemia among asymptomatic community members and that these carriers are highly infectious to mosquitoes (data not shown). In addition to the difficulty in harvesting a large number of eligible 
gametocyte carriers through surveillance of suspected malaria cases in rural health clinics, the infection rate and intensity in Anopheles gambiae mosquitoes with those eligible gametocyte carriers were very low. It is believed that the feeding technique through artificial membrane is not the reason for this low infection outcome since the direct membrane feeding assay represents a good proxy for the direct feeding when mosquitoes are allowed to feed directly on volunteers' skin $(35,36)$. In both cases, while the infectiousness of gametocytes to mosquitoes is highly variable, gametocyte density is thought to represent an important determinant for successful infections $(8,21)$. The low gametocyte density (range: 16-128 gametocytes per microliter) in eligible volunteers is probably the primary reason for the observed poor infectiousness in anopheline mosquitoes. The concomitantly presence of asexual parasites in gametocyte positive patients indicated that either they have been carrying the infection for more than a week before reporting to the health centre, or the current gametocytes may have originated from past infections. Therefore, the absence of gametocyte infectivity might further be explained by previous use of antimalarials, direct influence from specific immune factors(37-39) and possibly indirect impacts of toxins factors stimulated by the asexual parasites(40).

In conclusion, through surveillance of suspected malaria cases at outpatients at a rural health clinic in western Kenya, we found that relatively few patients are suitable gametocyte donors for experimental studies of vector competence. Within the experimental structures imposed by our protocol requirements and ethical considerations, the major observation is that our study failed to reproduce data similar to those produced elsewhere in Africa where P. falciparum infectivity has been extensively studied after selection of study subjects from clinically ill population $(8,11,12)$. Overall, transmission studies in clinically ill subjects have been variable and seldom sufficient to have much information on the infectiousness reservoir of infection. Our study is simply a contribution to this general knowledge, with particular reference to the situation in Western Kenya. Although rural health clinics in malaria-endemic areas represent excellent surveillance points for monitoring malaria infection dynamics and evaluation of the effects of malaria intervention programmes, acquirement of gametocyte-infected blood for vector competence studies requires alternative approaches such as active surveillance in the community and schools $(10,20,21,37)$ or follow up of patients post antimalarial drug treatment $(12-25,26,29)$.

\section{ACKNOWLEDGEMENTS}

To W. Onyango and A. Ouko of the Mbita Health Centre, Suba district, A. Githeko at Kenya Medical Research Institute (KEMRI), L. Omukuba, H. Akelo, P. Ongele, J. Arija and B. Njiru at the Mbita Point
Research Training Center of ICIPE, and all the patients for their support, assistance and cooperation. We also thank E. Walczak for critical comments on the manuscript and for expert statistical advice. This paper is submitted for publication with the approval of the Director General of ICIPE. This study was supported by grants from National Institutes of Health (U19 A145511, D43 TW01142, TW00920 and TW 01505), ICIPE ARPPIS scholarships and the Swiss National Science Foundation (Project ID. 31-59380.99).

\section{REFERENCES}

1. Alles, H.K., Mendis K.N. and Carter, R. Malaria mortality rates in South Asia and in Africa: Implications for malaria control. Parasitology Today. 1998; 14:369-375.

2. Snow, R.W., Craig, M., Deichmann, U. and Marsh, K. Estimating mortality, morbidity and disability due to malaria among Africa's non-pregnant population. Bull. World Hlth. Org. 1999; 77:624-640.

3. Breman, J.G. The ears of the hippopotamus: manifestations, determinants, and estimates of the malaria burden. Amer. J. Trop. Med. Hyg. 2001; 64:1-11.

4. Kirigia, J.M., Snow, R.W., Fox-Rushby, J. and Mills, A. The cost of treating paediatric malaria admissions and the potential impact of insecticide-treated nets on hospital expenditure. Trop. Med. Intern. Hlth. 1998; 3:145-150.

5. Ruiz, W. and Kroeger, A. The socioeconomic impact of malaria in Colombia and Ecuador. Hlth. Pol. Plann. 1994; 9:144-154

6. Gallup, J.L., and Sachs, J.D. Malaria, climate and poverty. Cambridge: Harvard Institute for International Development. 1999.

7. Beier, J.C., Killeen, G.F. and Githure, J. Short report: Entomologic inoculation rates and Plasmodium falciparum malaria prevalence in Africa. Amer. J. Trop. Med. Hyg.. 1999; 61:109-113.

8. Tchuinkam, T., Mulder, B., Dechering, K., et al. Experimental infections of Anopheles gambiae with Plasmodium falciparum of naturally infected gametocyte carriers in Cameroon: Factors influencing the infectivity of mosquitoes. Trop. Med. Parasit. 1993; 44:271-276.

9. Toure, Y.T., Doumbo, O., Toure, A., et al. Gametocyte infectivity by direct mosquito feeds in an area of seasonal malaria transmission: implications for Bancoumana, Mali as a transmission-blocking vaccine site. Amer. J. Trop. Med. Hyg. 1998; 59:481-486.

10. Muirhead-Thomson, R.C. Factors determining the true reservoir of infection of Plasmodium falciparum and Wuchereria bancrofti in a west African village. Trans. Royal Soc. Trop. Med. Hyg. 1954; 48:208-224.

11. Smalley, M.E., Brown, J., and Bassett, N.M. The rate of production of Plasmodium falciparum gametocytes during natural infections. Transactions of the Royal Society of Tropical Medicine and Hygiene. 1981; 75:318-319.

12. Robert, V., Awono-Ambene, H.P., Le Hesran, J.Y., and Trape, J.F. Gametocytemia and infectivity to mosquitoes of patients with uncomplicated Plasmodium falciparum malaria attacks treated with chloroquine or sulfadoxine plus pyrimethamine. Amer. J. Trop. Med. Hyg. 2000; 62:10-16.

13. Drakeley, C.J., Akim, N.I., Sauerwein, R.W., Greenwood, B.M., and Targett, G.A. Estimates of the infectious reservoir of Plasmodium falciparum malaria in The Gambia and in Tanzania. Trans. Royal Soc. Trop. Med. Hyg. 2000; 94:472476.

14. Beier, J.C., Perkins, P.V., Onyango, F.K. et al. Characterization of malaria transmission by Anopheles (Diptera: Culicidae) in Westem Kenya in preparation for malaria vaccines trials. $J$. 
Med. Entom. 1990; 27:570-577.

15. Haji, H., Smith, T., Charlwood, J. D., and Meuwissen, J.H. Absence of relationships between selected human factors and natural infectivity of Plasmodium falciparum to mosquitoes in an area of high transmission. Parasitology. 1996; 113:425431.

16. Mendis, K.N., David, P.H., and Carter, R. Human immune responses against sexual stages of malaria parasites: considerations for malaria vaccines. Intern. J. Parasit. 1990; 20:497-502.

17. Miller, L.H., Howard, R.J., Carter, R., and Good, M.F. Research toward malaria vaccines. Science. 1986; 234:13491356.

18. Minakawa, N., Githure, J.I., Beier, J.C., and Yan, G. Anopheline mosquito survival strategies during the dry period in western Kenya. J. Med. Entom. 2001; 38:388-392.

19. Minakawa, N., Mutero, C.M., Githure, J.I., Beier, J.C., and Yan, G. Spatial distribution and habitat characterization of Anopheline mosquito larvae in Western Kenya. Amer. J. Trop. Med. Hyg. 1999; 61:1010-1016.

20. Githeko, A.K., Brandling-Bennett, A.D., Beier, M., et al. The reservoir of Plasmodium falciparum malaria in a holoendemic area of western Kenya. Trans. Royal Soc. Trop. Med. Hyg. 1992; 86:355-358.

21. Boudin, C., Olivier, M., Molez, J.F., Chiron, J.-P., and Ambroise-Thomas, P.A. High human malarial infectivity to laboratory-bred Anopheles gambiae in a village in Burkino Faso. Amer. J. Trop. Med. Hyg. 1993; 48:700-706.

22. Benedict, M.Q. Care and maintenance of anopheline mosquito colonies. Crampton J. M, Beard, C.B., Louis, C. eds. Molecular biology of insect disease vectors: A methods manual. New York: Chapman and Hall. 1997; 3-12.

23. Smith, T., Charlwood, J.D., Kihonda, J., et al. Absence of seasonal variation in malaria parasitemia in an area of intense seasonal transmission. Acta Tropica. 1993; 54:55-72.

24. Snow, R.W., Nahlen, B., Palmer, A., Donnelly, C.A., Gupta, S., and Marsh, K. Risk of severe malaria among African infants: Direct evidence of clinical protection during early infancy. J. Infect. Dis. 1998; 177:819-822.

25. Bousema, J.T., Gouagna, L.C., Meutstege, et al. Treatment failure of pyrimethamine-sulphadoxine and induction of Plamodium falciparum gametocytaemia in children in westem Kenya. Trop. Med. Intern. Hlth. 2003; 8:427-430.

26. Mberu, E.K., Mosobo, M.K., Nzila, A.M., Kokwaro, G.O., Sibley, C.H. and Watkins, W.M. The changing in vitro susceptibility pattern to pyrimethamine/sulphadoxine in Plasmodium falciparum field isolates from Kilifi, Kenya. Amer. J. Trop. Med. Hyg. 2000; 62:396-401.

27. Mutero, C.M., Ouma, J.h., Agak, B.K., Wanderi, J.A. and Copeland, and R.S. Malaria prevalence and use of selfprotection measures against mosquitoes in Suba district. East Afr. Med. J. 1998; 75:11-16.
28. Shute, P.G., and Maryon, M. A study of gametocytes in a West African strain of Plasmodium falciparum. Trans. Royal So. Trop. Med. Hyg. 1951; 44:421-438.

29. Akim, N.I., Drakeley, C., Kingo, T., Simon, B., Senkoro, K., and Sauerwein, R.W. Dynamics of Plasmodium falciparum gametocytemia in symptomatic patients in an area of intense perennial transmission in Tanzania. Amer. J. Trop. Med. Hyg. 2000; 63:199-203.

30. Sinden, R.E. The cell biology of sexual development in Plasmodium. Parasitology. 1983; 86:7-28.

31. Price, R., Nosten, F., Simpson, J.A., et al. Risk factors for gametocyte carriage in uncomplicated falciparum malaria. Ame. J. Trop. Med. Hyg. 1999; 60:1019-1023.

32. Carter, R., and Miller, L.H. Evidence for environmental modulation of gametocytogenesis in Plasmodium falciparum in continuous culture. Bull. World Hlth Organ. 1979; 57:3752.

33. Schneweis, S., Maier, W.A., and Seitz, H.M. Haemolysis of infected erythrocytes-a trigger for formation of Plasmodium falciparum gametocytes? Parasitological Research. 1991; 77:458-460.

34. Puta, C., and Manyando, C. Enhanced gametocyte production in Fansidar-treated Plasmodium falciparum malaria patients: implications for malaria transmission control programmes. Trop. Med. and Inter. Health. 1997; 2:227-229.

35. Awono-Ambene, H.P., Diawara, L., and Robert, V. Comparison of direct and membrane feeding methods to infect Anopheles arabiensis with Plasmodium falciparum. Amer. J. Trop. Med. Hyg. 2001; 64:32-34.

36. Bonnet, S., Gouagna, C., Safeuki, I., Meunier, J.-Y., and Boudin, C. Comparison of artificial membrane feeding with direct skin feeding to estimate infectiousness of Plasmodium falciparum gametocyte carriers to mosquitoes. Trans. Royal Soc. Trop. Med. Hyg. 2000; 94:103-106.

37. Graves, P.M., Burkot, T.R., et al. Measurement of malarial infectivity of human populations to mosquitoes in the Madang area, Papua New Guinea. Parasitology. 1988; 96:251-263.

38. Vermeulen, A.N., Ponnudurai, T., Becker, P.J.A., et al. Sequential expression of antigens on sexual stages of Plasmodium falciparum accessible to transmission-blocking antibodies in the mosquito. J. Exper. Med. 1985; 162:14601476.

39. Ong, C.S.L., Zhang, K.Y., Eida, S.J., et al. The primary antibody response of malaria patients to Plasmodium falciparum sexual stage antigens which are potential transmission blocking vaccine candidates. Parasite Immunology. 1990; 120: 447456.

40. Naotunne, T.S., Karunaweera, N.D., Mendis, K.N., and Carter, R. Cytokine-mediated inactivation of malarial gametocytes is dependent on the presence of white blood cells and involved reactive nitrogen intermediates. Immunology. 1993; 78:555-562. 